\title{
Percepção das dificuldades relacionadas ao tratamento entre pessoas com hipertensão arterial sistêmica 1
}

Treatment related problems perception for people with systemic hypertension

\section{La percepción de las dificultades relacionadas con el tratamiento en personas con hipertensión}

\author{
Isabela Cristina Zagui $_{3}^{2}$ \\ Stefânia Ribeiro Borges 3 \\ Juliana Guisardi Pereira \\ Vanessa Alvarenga Pegoraro \\ Sebastião Junior Henrique Duarte ${ }^{6}$
}

\footnotetext{
${ }^{1}$ Trabalho de conclusão de curso em Enfermagem. Vinculado ao projeto de extensão: Programa de Educação pelo Trabalho para a Saúde/Saúde da Família em Várzea Grande/MT, com financiamento do Ministério da Saúde. Parte integrante da pesquisa: "Caracterização da clientela adscrita à Estratégia de Saúde da Família em Várzea Grande: implicações para intervenção em Saúde”, aprovado pelo Comitê de Ética em Pesquisa com Seres Humanos do Hospital Júlio Muller sob no890/CEP-HUJM/2010.
}

2 Acadêmica do $9^{\circ}$ semestre da Faculdade de Enfermagem da Universidade Federal de Mato Grosso. Rua Maringá, n. 111, Coophema, Cuiabá, MT, CEP 78085185. E-mail:isa.zagui @ hotmail.com 3 Acadêmica do $9^{\circ}$ semestre da Faculdade de Enfermagem da Universidade Federal de Mato Grosso. Rua Maringá, n. 111, Coophema, Cuiabá, MT, CEP 78085185. E-mail: faninha_ribeiro@yahoo.com.br 4 Enfermeira. Doutoranda em Ciências pela Escola de Enfermagem da Universidade de São Paulo. Professora Assistente. Faculdade de Enfermagem da Universidade Federal do Mato Grosso. Rua Vinte e Cinco de Agosto, n. 65 ap. 1502, Duque de Caxias, Cuiabá, MT, CEP 78043-382. tel.(65)8135-9033.E-mail: juguisardi@yahoo.com.br

5 Enfermeira. Especialista em Saúde do Trabalhador e Saúde da Família. Professora substituta. Faculdade de Enfermagem da Universidade Federal do Mato Grosso. Rodovia Emanuel Pinheiro.Avenida A.s/n. Residencial San Marino.Quadra 16, casa 23.Parque das Nações. Cuiabá. MT.CEP: 78.055-799.Tel:(65)81541944.E-mail: vanessapeg@yahoo.com.br

6Enfermeiro. Doutor em ciências. Professor Adjunto. Faculdade de Enfermagem da Universidade Federal do Mato Grosso. Rua José da Silva Monteiro, 168 apart 101, Bairro Miguel Sutil, CEP 78.048-295, Cuiabá-MT. Email: sjhd@usp.br 


\section{RESUMO}

Estudo de natureza descritiva, com abordagem qualitativa, que teve como objetivo conhecer a percepção de pessoas com Hipertensão Arterial Sistêmica (HAS), a respeito das dificuldades advindas com o tratamento de HAS. Participaram do estudo 7 pessoas com diagnóstico de hipertensão há pelo menos 5 anos, residentes em uma área de abrangência da Estratégia de Saúde da Família, município de Várzea Grande - MT. Os dados foram coletados por meio de entrevista semi-estruturada e individual. Utilizou-se o Discurso do Sujeito Coletivo para organização dos dados e a Teoria das Representações Sociais como referencial teórico. A análise se deu pela identificação do senso comum, evidenciando as representações sociais que ancoram os saberes, vivências e necessidades desses hipertensos que necessitam de atenção por parte dos profissionais de saúde. Os resultados evidenciaram que os hipertensos apresentam dificuldade para o tratamento medicamentoso, mudança no estilo de vida, medo das complicações decorrentes da HAS e que há deficiências no serviço de saúde. As considerações finais revelam a necessidade de articular ações que contribuam com a adesão ao tratamento da HAS, como melhorias na oferta do serviço prestado, co- responsabilização e estabelecimento de vínculo por parte da equipe de saúde, com reflexo na qualidade de vida dos usuários.

Descritores: Saúde do adulto; Hipertensão; Pesquisa qualitativa; Discurso do Sujeito Coletivo.

\section{ABSTRACT}

This descriptive study with qualitative approach aimed to assess the perception of people with high blood pressure (hypertension), about the difficulties stemming from the treatment of hypertension. The study included seven individuals diagnosed with hypertension for at least 5 years, living in an area covered by the Family Health Strategy, in Várzea Grande city - MT. Data were collected through semi-structured individual interviews. We used the Collective Subject Discourse to organize the data and the theory of social representations as theoretical. The analysis was done by identifying common sense, highlighting the social representations that anchor the knowledge, experiences and needs of hypertensive patients in need of attention from health professionals. The results "showed that hypertensive patients have difficulty to drug treatment, change in lifestyle, fear of complications of hypertension and that there are deficiencies in the health service. The final considerations reveal the need for joint actions that contribute to adherence to treatment of hypertension, such as improvements in the provision of service, co-responsibility and establishing a relationship on the part of the healthcare team to reflect in the users' quality of life.

Descriptors: Adult Health; Hypertension; Qualitative Research; Discourse of Collective Subject.

\section{RESUMEN}

Revista Eletrônica Gestão \& Saúde • Vol.02, No. 01, Ano 2011 • p. 123-134 
Estudio descriptivo con enfoque cualitativo que tuvo como objetivo conocer la percepción de las personas con presión arterial alta (hipertensión), acerca de las dificultades derivadas del tratamiento de la hipertensión. El estudio incluyó a siete personas con diagnóstico de hipertensión arterial por lo menos 5 años, que viven en un área cubierta por la Estrategia de Salud de la Familia, la ciudad de Várzea Grande - MT. Los datos fueron recolectados a través de semi-estructuradas individuales. Se utilizó el Discurso del Sujeto Colectivo para organizar los datos y la Teoría de las Representaciones Sociales como teórico. El análisis tuvo en la identificación de lo sentido común, las representaciones sociales que muestran que anclan el conocimiento, experiencias y necesidades de los pacientes hipertensos que requieren la atención de profesionales de la salud. Los resultados mostraron que los hipertensos tienen dificultades en el tratamiento de drogas, cambios en el estilo de vida, el miedo a las complicaciones de la hipertensión y que hay deficiencias en los servicios de salud.Las observaciones finales de relieve la necesidad de articular acciones que contribuyan a la adherencia al tratamiento de la hipertensión arterial, tales como mejoras en la prestación del servicio, la responsabilidad y co-establecimiento de las relaciones del equipo de salud, que se refleja en la calidad de vida de los usuarios.

Descriptores: Salud del adulto; Hipertensión; Investigación cualitativa; Discurso del Sujeto Colectivo.

\section{I - Introdução}

As doenças e os agravos não transmissíveis (DANTs) têm configurado como uma das principais causås de morbimortalidade no mundo, responsáveis por cerca de $60 \%$ dos óbitos anuais e $46 \%$ da carga global de doença, sendo predominante principalmente nos países industrializados e nos emergentes ${ }^{(1-6)}$.

No Brasil, as DANTs seguem padrões semelhantes, constituindo em 2009 a principal causa de óbito no país, destacando-se, dentre elas, as doenças do aparelho circulatório e a Hipertensão Arterial Sistêmica (HAS) como principais fatores de risco para as complicações, tais como o acidente vascular cerebral, infarto agudo do miocárdio e doença renal crônica ${ }^{(2)}$.

Estima-se que a prevalência atual da HAS no país chegue a $35 \%$ da população com idade maior que 40 anos, o que corresponde a 17 milhões de brasileiros hipertensos, constituindo-se num importante problema de saúde pública no Brasil e no mundo, com elevados custos médico, socioeconômico e psicossocial decorrentes principalmente das complicações ${ }^{(3,4)}$. 
Como condição crônica, a HAS requer mudanças no estilo de vida e precauções ao longo da vida, envolvendo estratégias para lidar com os sintomas e as relações familiares e sociais; longo tempo de cuidado, uma vez que o processo de cura é praticamente inexistente, gerando incapacidade residual e, algumas vezes, a recorrência da doença ${ }^{(5)}$.

A partir da classificação de risco individual e dos níveis pressóricos encontrados, duas abordagens terapêuticas para a HAS podem ser indicadas: o tratamento baseado em modificações no estilo de vida - que compreendem controle do peso incentivo a atividades físicas, alimentação saudável, abandono do tabagismo - e o tratamento medicamentoso ${ }^{(6)}$.

Como parte fundamental do tratamento, as modificações no estilo de vida envolvem mudanças estruturais por parte do hipertenso, podendo constituir-se num dos motivos que implica na baixa adesão ao tratamento. Outrossim, a HAS pode apresentar-se assintomática e o desconhecimento do diagnóstico pode levar a complicações antes do estabelecimento da terapêutica $^{(3)}$.

Considerando que a compreensão e a percepção que o usuário tem sobre a HAS constituem fator determinante sobre a melhora da adesão é que o presente estudo teve por objetivo conhecer a percepção de pessoas com HAS residentes na área de abrangência de uma Unidade de saúde da Família situada no município de Várzea Grande - MT, acerca das dificuldades relacionadas ao tratamento da HAS.

Essa busca se deu através do estudo do senso comum que permitiu apreender o modo como os hipertensos percebem e lidam com o tratamento, trazendo subsídios para o atendimento em saúde prestådo a essa população, podendo posteriormente discutir estratégias que venham a contribuir com as necessidades de saúde dessas pessoas.

\section{II - Metodologia}

Trata-se de um estudo de natureza descritiva, com abordagem qualitativa, realizado com hipertensos residentes na área de abrangência da Estratégia Saúde da Família (ESF) do bairro Manga, localizada no município de Várzea Grande-MT.

Participaram do estudo 7 pessoas das 18 que participavam da reunião de grupo de hipertensão arterial no momento da coleta dos dados, tendo como critério de inclusão ter o diagnóstico há pelo menos 5 anos, sendo excluídos aqueles que tivessem outras patologias 
crônicas associadas. As reuniões de grupo de hipertensos ocorrem semanalmente e a média de participantes é de 20 pessoas.

Para coleta dos dados foi utilizada a técnica de entrevista semi-estruturada e individual, que foram gravadas em fita K-7, posteriormente transcritas e armazenadas em CDROM.

Adotou-se a teoria das Representações Sociais (RS) como referencial teórico e o Discurso do Sujeito Coletivo (DSC) para organização e análise dos dados.

As RS são definidas como sendo uma forma de conhecimento socialmente elaborado e compartilhado, podendo ainda ser definidas como modalidades de conhecimento prático, orientadas para comunicação e para a compreensão do contexto social, que contribui .na construção de uma realidade comum a um conjunto social. Elas são equivalentes aos mitos e crenças das sociedades, ou seja, podendo ser uma versão contemporânea do senso comum. Recomenda-se que a RS seja estudada articulando-se elementos afetivos, mentais e sociais ${ }^{(7-}$ 8).

A técnica do DSC consiste numa forma para a organização, tabulação e leitura dos dados qualitativos de origem verbal, onde permite a construção do pensamento coletivo, de forma a revelar a maneira como naturalmente as pessoas pensam, oportunizando acesso a dados subjetivos da realidade, onde manifestam seu posicionamento diante do assunto abordado, tratando-se de um compartilhamento de ideias dentro de um grupo social ${ }^{(9)}$.

Para a formulação dos DSC foram utilizados trechos das entrevistas, sendo transcrições literais dos depoimentos, que permitiram resgatar a essência e o essencial das falas, denominadas como expressões-chave. Obteve-se as ideias centrais a partir de recortes significativos dos depoimentos, ou seja, as palavras ou expressões linguísticas que mostram, de maneira precisa e sintetizada, o sentido presente. As expressões-chave que exemplificam as ideias centrais semelhantes foram arranjadas de forma lógica e coerente, compondo um único discurso escrito na primeira pessoa do singular formando, assim, o Discurso do Sujeito Coletivo ${ }^{(10)}$.

No presente estudo, as questões formuladas, por sua própria natureza, não geraram conteúdos discordantes, razão pela qual ocorre a regularidade nos discursos. A representatividade das falas foram identificadas por meio da emissão desses conteúdos nos discursos dos sujeitos, denominados por pseudônimos: Flor de Laranjeira, Girassol, Hortência, Orquídea, Rosa Amarela, Rosa Branca e Rosa Itália. 
A pesquisa, em conformidade com a Resolução 196/96 sobre Pesquisa Envolvendo Seres Humanos do Conselho Nacional de Saúde, seguiu rigorosamente os procedimentos metodológicos e éticos, sendo aprovado pelo Comitê de Ética em Pesquisa do Hospital Universitário Júlio Muller da Universidade Federal de Mato Grosso, sob o $n^{\circ}$ 890/CEPHUJM/10. Todos os entrevistados foram orientados e esclarecidos sobre a pesquisa e assinaram o Termo de Consentimento Livre e Esclarecido.

Este estudo está vinculado ao Programa de Educação pelo Trabalho para a Saúde (PET-Saúde) Saúde da Família, constituído numa parceria entre instituições de ensino da Universidade Federal do Mato Grosso e a Secretaria Municipal de Saúde de Várzea GrandeMT, financiado pelo Ministério da Saúde ${ }^{(11)}$.

\section{III - Resultados}

A faixa etária dos 7 entrevistados estava entre 43 e 63 anos, sendo 1 do sexo masculino e 6 do sexo feminino. Quanto a raça, 3 se denominaram de cor parda, 3 de cor preta e 1 branca. Com relação a escolaridade, 3 pessoas eram analfabetas, 1 tinha ensino fundamental incompleto, 1 ensino fundamental completo e 2 com ensino médio completo. Em relação ao tempo de diagnóstico, 2 tinham há menos de 10 anos e 5 com mais de 10 anos.

Para a obtenção dos conteúdos de fala, foram solicitadas as seguintes perguntas: "Desde que você descobriu que tem pressão alta, alguma coisa mudou na sua vida? O quê?", "Você tem alguma dificuldade para fazer o tratamento de pressão alta?", e "Na sua opinião, o que seria necessário para facilitar seu tratamento?"

$\mathrm{Na}$ análise das entrevistas foi possível encontrar as quatro Ideias Centrais (IC) que compuseram os discursos a seguir:

\section{IDÉIA CENTRAL 1 - Esquecimento na tomada da medicação}

Não lembro toda hora de tomar o remédio, não é todo dia. Às vezes não tomo o remédio nas horas certas, mas tomo todo dia. Minha mente não é muito boa; eu sou muito esquecida, aí eu não tomo medicamentos na hora certa. Por que às vezes vou tomar, aí vou fazer outra coisa e esqueço aquele medicamento, aí se tenho que ir em algum lugar já não tomo o remédio. Às vezes quando eu esqueço de tomar o remédio a pressão dá uma subida. O problema é eu 
lembrar. Eu tomo, mas agora esses dias atrás eu tenho esquecido... aí dá uma subida. E se ta meio altinha é porque já passei da hora de tomar o remédio. Minha filha telefona do serviço dela, né? E fala: "Mamãe, toma remédio! Mamãe, toma remédio!

\section{IDÉIA CENTRAL 2 - Existem falhas na disponibilidade das medicacões na rede básica}

Eu não vou lá [na Unidade de Saúde] pra pegar o remédio, aí tem que ir meu filho, que tem 9 anos ou meu marido. Aí quando vai, às vezes não tem o remédio, né. Não tem todos os remédios; aí tem que comprar. Aí a dificuldade era que a gente pegava no posto e agora quase não tem mais, aí tem que comprar. Porque se não tem medicamento no posto, eu vou na farmácia popular. O negócio é o governo não deixar faltar o remédio. Eles falam melhorar, melhorar saúde e deixa faltar remédio, eles não deviam deixar faltar né?

\section{IDÉIA CENTRAL 3 - Medo de complicações relacionadas a HAS}

A gente com pressão alta sempre tem problema. Eu tive derrame, sentia tontura, bastante! Depois que eu tive o derrame, tinha que tomar o remédio direto, né? É só tomar o remédio, porque se num tomar aí é perigoso me dar um derrame. A pressão sobe e me dá um derrame, né? Me dá um infarto e aí pronto, o corpo já pulou, né? Então tem que tomar pra controlar.

\section{IDÉIA CENTRAL 4 - Mudancas no estilo de vida}

[Minha vida] mudou, eu não posso comer certas coisas, eu não posso ficar nervosa. Eu não posso tomar muito sol, não posso andar muito rápido, né. Agora, de uns tempos pra cá, qualquer coisa que, se eu ficar nervosa ela sobe na hora. Eu sou muito sentimental, agora se eu não tiver nervosa a pressão é 12 por 8 . Agora eu to com menino aí que me ajuda, mas eu como pouco sal, eu recomendo pouco sal, pouca gordura, assim. Não durmo direito. Esse que é o problema de não comer e não dormir, né? Porque o dia que a pressão tá alta a gente não dorme. Com a pressão alta dá mais cansaço conforme você vai fazendo no dia. Também tive que começar a tomar remédio.

\section{IV - Discussão}


O estudo evidenciou que os discursos dos hipertensos apontam para uma importante preocupação com o tratamento medicamentoso da HAS, evidenciando estar ancorados numa perspectiva mais curativa do que de promoção da saúde e prevenção das complicações, como por exemplo, através da adoção de hábitos saudáveis. Essa perspectiva pode ser um reflexo do modelo biomédico de atenção à saúde que se tornou hegemônico em nosso meio, centralizado no profissional de saúde, tendo o usuário um papel passivo, com pouca oportunidade de compartilhar ou participar nas decisões que se referem ao seu corpo e a sua saúde ${ }^{(12)}$.

Os discursos apontaram uma dificuldade relacionada ao tratamento medicamentoso, que é a administração inadequada pelo esquecimento de tomá-los na regularidade prescrita; de forma que não os tomam diariamente nem nos horários recomendados, podendo vir a comprometer a eficácia do tratamento. Esse esquecimento pode ser devido à carga de atividades diárias, sejam domésticas ou fora do domicílio, bem como pelo fato de a HAS se apresentar muitas vezes de forma assintomática, só se lembram de tomá-la quando sentem algum sintoma que julgam ser da pressão alta.

Num estudo sobre adesão ao tratamento entre hipertensos, os autores ${ }^{(13)}$ também identificaram o esquecimento como motivo para deixar de tomar os medicamentos, mesmo tendo se identificado o conhecimento dos hipertensos sobre a cronicidade e gravidade da HAS; necessidade de tratamento por toda vida; ocorrência de complicações quando não tratada; ausência de sintomatologia específica e valores da pressão que caracterizam hipertensão. Asssim sendo, os autores entendem o esquecimento como uma postura negativa que pode denotar pouco envolvimento do paciente com o seu problema de saúde.

A literatura ${ }^{(14)}$ indica que uma possibilidade de conseguir melhorias nesse sentido seria substituir medicamentos regulares que necessitam de mais de uma tomada diária por outros de liberação lenta e dose única, o que reduziria a frequência de tomada de medicamento ao longo do dia.

Outra ideia central que surgiu foi o medo das complicações decorrentes da HAS como, por exemplo, acidente vascular cerebral e infarto agudo do miocárdio, também associadas ao uso das medicações de forma a preveni-las, o que denota conhecimento sobre complicações da HAS enquanto condição crônica.

Ainda com relação à medicação, os hipertensos apontaram falhas na disponibilização dos mesmos na rede básica, levando à necessidade de comprá-ló com seus próprios recursos, 
que muitas vezes não é possível, essa situação contribui à não continuidade do tratamento medicamentoso corretamente até que a medicação esteja disponível na unidade de saúde, acarretando nesse período a elevação da pressão e a possibilidade de desencadeamento de alguma complicação.

Diante disso, concordamos com alguns altores ${ }^{(15)}$ ao afirmarem que a promoção da saúde não se restringe somente como responsäbilidade ao setor saúde, mas de uma relação entre os diversos setores do governo municipal, estadual e federal, os quais articulam políticas e ações que tragam melhoria das condições de vida da população e da oferta de serviços essenciais que produzam bem-estar ao seres humanos. É importante considerar ${ }^{(4)}$, que a adesão tem um caráter multidimensional, ou seja, não depende unicamente da pessoa cóm hipertensão, pois envolve diferentes aspectos, tais como os profissionais e o sistema de saúde, tornando-se necessária a ação conjunta para que a adesão seja alcançada.

A representação dos hipertensos sobre a mudança do seu estilo de vida indica a necessidade dos indivíduos manterem seu dia-a-dia com mínimo possível de estresse, por acreditar que pode influenciar na elevação da pressão arterial, e como consequência interferir no seu estado de bem estar, ao referirem insônia e cansaço. O estresse e emoções fortes podem ser um dos elementos desencadeadores de reatividade cardiovascular, que se colaboram nas mudanças de pressã̃o arterial ou frequência cardíaca que acontecem respondendo a uma situação ou evento específico ${ }^{(16)}$.

No que refere à alimentação, os discursos revelaram um esforço no sentido de evitar sal e gordura. Sabendo que mudar hábitos alimentares não é uma tarefa fácil, o processo de adaptação é lento e longo, pois cada indivíduo tem uma cultura e forma diferente de viver antes do diagnóstico da HAS, o que deve levar o profissional de saúde a ter paciência, habilidade e persuasão, sabendo que essa medida estará colaborando para controlar a pressão arterial e prevenindo o aparecimento das complicações ${ }^{(17)}$.

Notou-se, de forma geral, que há conhecimento por parte dos hipertensos envolvidos nesse estudo sobre a hipertensão, especialmente quanto à importância de tomar a medicação para o controle da hipertensão arterial na frequência e horários determinados, evitando-se as complicações; cuidados com a alimentação e com o controle do estresse. Entretanto ter conhecimento sobre a doença e tratamento muitas vezes não é garantia de controle da HAS, demonstrando uma diferença essencial entre conhecimento e adesão ${ }^{(13)}$. Para os autores, o 
conhecimento é racional, enquanto a adesão é um processo complexo que envolve fatores biossociais, emocionais e barreiras concretas.

Para que a assistência à pessoa com hipertensão seja prestada com qualidade é preciso conhecer as dificuldades advindas com o tratamento desse agravo e que a equipe de saúde da unidade de saúde da família, principalmente enfermeiros e médicos, incorporarem a percepção do paciente acerca da sua doença em seus cuidados. Essa postura, que diverge do modelo biomédico, aponta para a necessidade de profissionais sensíveis à realidade paciente, dispostos a ouvir suas queixas e encontrar, conjuntamente, estratégias que o auxiliem na adaptação ao estilo de vida influenciado pela doença" (18).

\section{V - Considerações finais}

Os discursos evidenciaram que às Representações Sociais das pessoas com HAS participantes do estudo são marcadas por sentimentos negativos, especialmente no que se refere à dificuldade para lembrar a medicação, mudanças no estilo de vida que desencadeiam sentimentos negativos, o medo das possíveis complicações pelo agravo, e as deficiências na disponibilidade das medicações na rede básica.

O estudo deixa claro que é necessário articular ações para aumentar a adesão ao tratamento, com isso a melhora na qualidade de vida das pessoas com HAS. Tais ações compreenderiam melhorias na oferta do serviço, principalmente no que se refere a distribuição gratuita e aces̊so aos medicamentos, aproximação do serviço de saúde aos usuários, tendo em vista os princípios de corresponsabilização e vinculo requeridos na Estratégia Saúde da Família e também orientações contínuas e apoio psicológico a pessoa com hipertensão e seus familiares.

\section{Referências}

1 LESSA, I. Doenças crônicas não-transmissíveis no Brasil: um desafio para a complexa tarefa da vigilância. Ciência \& Saúde Coletiva, v.9, n.4, p. 931-943, 2004. Disponível em: http://www.scielo.br/pdf/csc/v9n4/a14v9n4.pdf.

2 DATASUS- "Datasus - Centro Tecnológico de Informação e Informática do SUS". Disponível em http://www.datasus.gov.br. Acesso em setembro de 2009. 
3 BRASIL; Ministério da Saúde. Secretaria e Atenção à Saúde - Departamento de Atenção Básica. Cadernos de Atenção Básica - Hipertensão arterial sistêmica para o Sistema Único de Saúde. Brasília, p.58; Série A. ISBN 85-334-1189-8, 2006.

4 DOSSE C. et al. Fatores associados a não-adesão dos pacientesao tratemento da hipertensão arteial. Revista Latino-Americana de Enfermagem, Ribeirão Preto, v. 17, n. 2, abril, 2009.

5 FREITAS, M.C; MENDES, MMR. Condição crônica: análise do conceito no contexto da saúde do adulto. Rev Latino-am Enfermagem; Ribeirão Preto, v.15, n.4, jul-ago, 2007.

6 WORLD HEALTH ORGANIZATION (WHO). Adherence to long-term therapies: evidence for action. Geneva: WHO; 2003.

7 MOSCOVICI, S. Representações sociais: investigações em psicologia social. Petrópolis: Vozes, 2003.

8 JODELET, D. Representações sociais: um domínio em expansão. Rio de Janeiro: Eduerj, 2002.

9 LEFRÉVE, F., LEFRÉVE A.M.C, TÉIXEIRA J.J.V, organizadores. O discurso do sujeito coletivo: uma nova abordagem metodológica na pesquisa qualitativa. EDUCS. Caxias do Sul, 2000.

10 DUARTE, S.J.H; ANDRADE, S.M.O. O Significado do pré-natal para mulheres Grávidas: uma experiência no município de Campo Grande, Brasil. Saúde Sociedade. São Paulo, v.17, n.2, p.132-139, 2008.

11 BRASIL. Portaria Interministerial n. 421, de 3 de março de 2010. Dispõem sobre o Programa de Educação pelo Trabalho para a Saúde (PET Saúde) e dá outras providências. Diário Oficial da União, Brasília, 5 mar. 2010. Seção 1, p. 43.

12 REINERS, A.A.O., NOGUEIRA M.S. Conscientização do usuário hipertenso para a adesão ao tratamento. Rev Latino-am Enfermagem 2009 janeiro-fevereiro; 17(1).

13 JESUS E.S, AUGUSTO M.A.O, GUSMÃO J., JÚNIOR D.M., ORTEGA K., PIERIN A.M.G. Perfil de um grupo de hipertensos: Aspectos biossociais, conhecimentos e adesão ao tratamento. Revista Acta Paul Enfermagem, v.21, n.1, p.59-65, 2008.

14 SARQUIS, L.M.M. et al. A adesão ao tratamento na hipertensão arterial: análise da produção científica. Rev.Esc.Enf.USP, v.32, n.4, p. 335-53, dez. 1998.

15 MACHADO, M.F.A.S., MONTEIRO, E.M.L.M, QUEIROZ, D.T, VIEIRA, N.F.C,. Integralidade, formação de saúde, educação em saúde e as propostas do SUS - uma revisão conceitual. Ciência \& Saúde Coletiva, 12(2), 2007, p.335-342. 
16 LIPP, M.E.N, FRARE, A., SANTOS, F.U. Efeitos de variáveis psicológicas na reatividade cardiovascular em momentos de stress emocional. Estudos de Psicologia. v. 2, n. 24, p. 161167. Campinas, 2007.

17 CESARINO,C. B.; CARDOSO, S.S.; MACHADO, M.R; BRAILE, D.M.; GODOY, M.F. Abordagem educativa sobre restrição salina ao paciente hipertenso. Arquivo Ciência e Saúde, v.11,n.4, p.234-7, 2004.

18 CAPRARA, A.; RODRIGUES, J. A relação assimétrica médico-paciente: repensando o vínculo terapêutico. Ciência e Saúde Coletiva, Rio de Janeiro, v. 9,n.1, p. 139-146, 2004. 